\title{
OXIDATIVE DEAROMATIZATION: ONE POT SYNTHESIS OF CHIRAL SPRIOIMIDAZOLIDINONE CYLOHEXADIENONES FROM AMINO ACID CHIRONS
}

ACS Meeting, Philadelphia March 26th, 2020

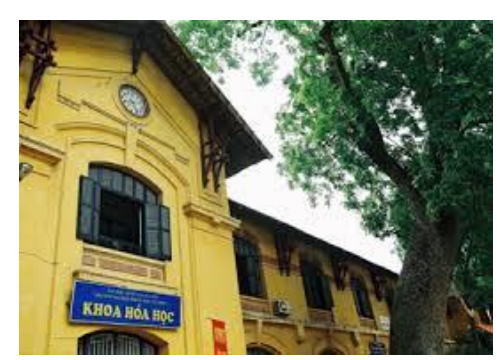

DUNG T. DO 


\section{Chiral pool synthesis}

- Chiral pool synthesis is a strategy that aims to improve the efficiency of enantioselective synthesis.

- The synthesis of a complex enantioenriched molecule from a stock of readily available enantiopure substances.

- Common chiral starting materials include monosaccharides and amino acids.

- The built-in chirality is preserved throughout reaction sequence. 


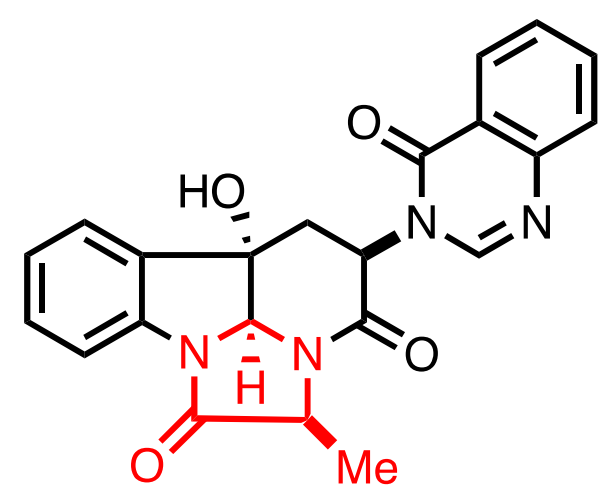

(-)Chaetominine

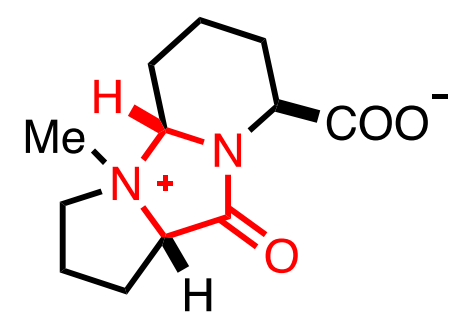

(-)-Dysibetaine PP<smiles></smiles>

Lead molecule

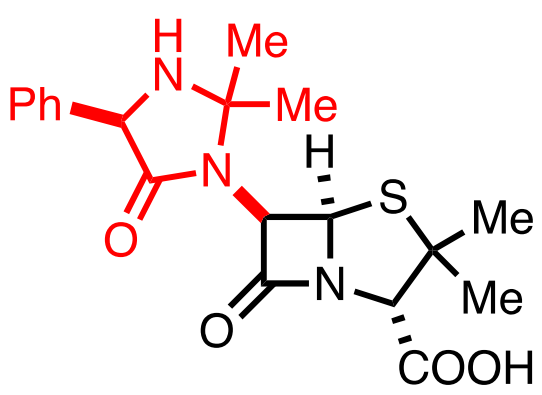

Hetacillin

J. Med. Chem. 2015, 58, 20, 8154-8165 


\section{Photoredox Catalysis with Organocatalysis}

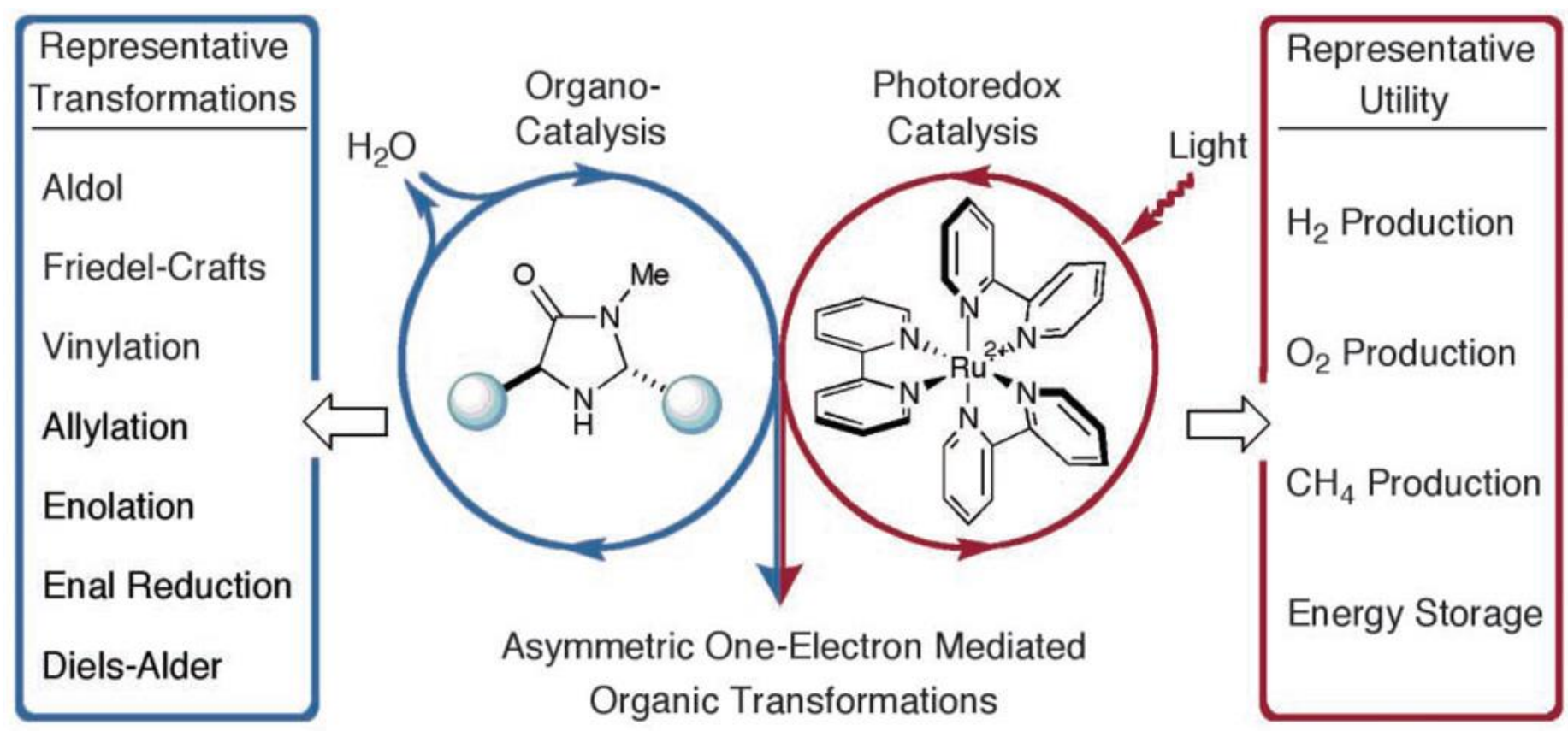

Enantioselective Catalytic Carbonyl $\alpha$-Alkylation<smiles>O=CCP</smiles>

aldehyde<smiles>[Ca]CBr</smiles>

alkyl bromide

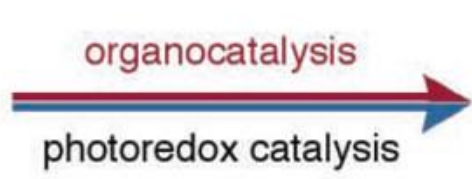

photon source<smiles>O=CC(P)CCl</smiles>

enantioenriched $\alpha$-alkylated aldehyde

Science, 2008, 322, 77-80 


\section{Synthesis of Imidazolidinone: Condensation}
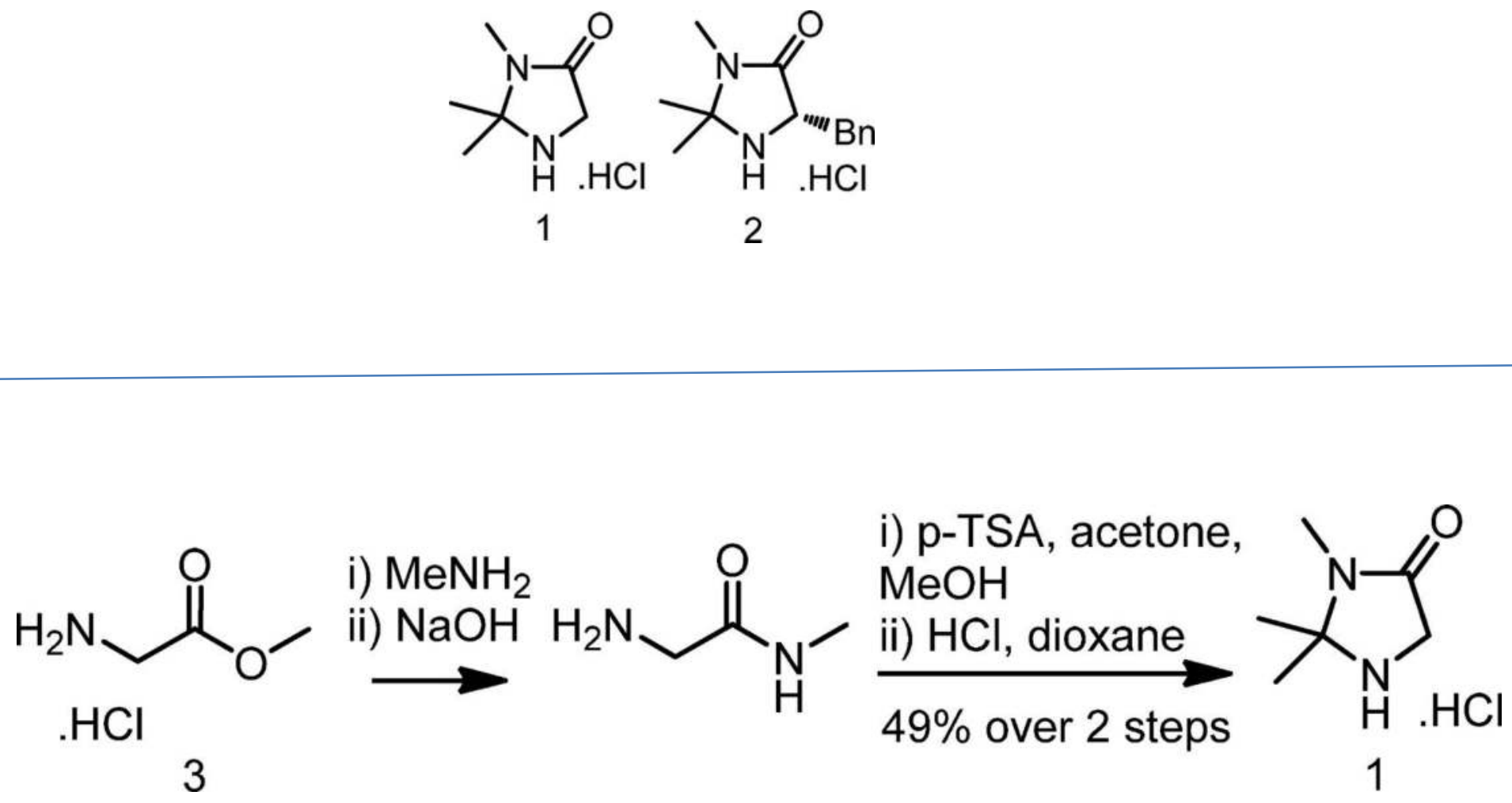

J. Am. Chem. Soc., 2000, 122 (17), 4243-4244

J. Chem. Educ., 2016, 93 (9), 1626-1630 
The Asymmetric Reduction of Imidazolinone
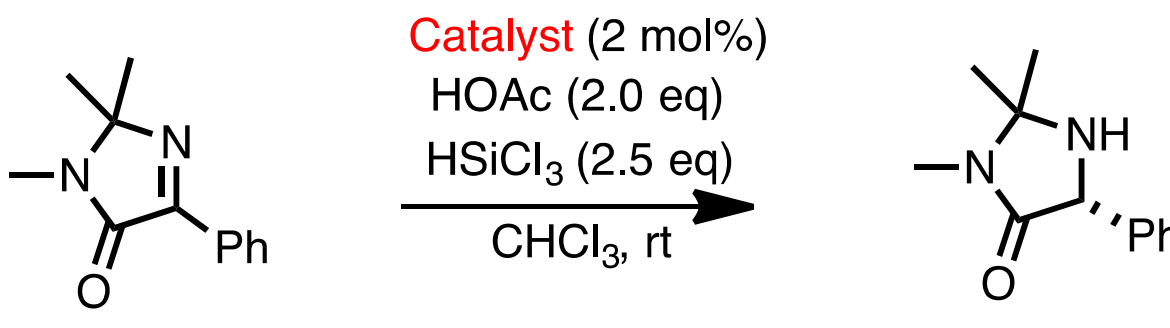

99\% yield

$94 \%$ ee

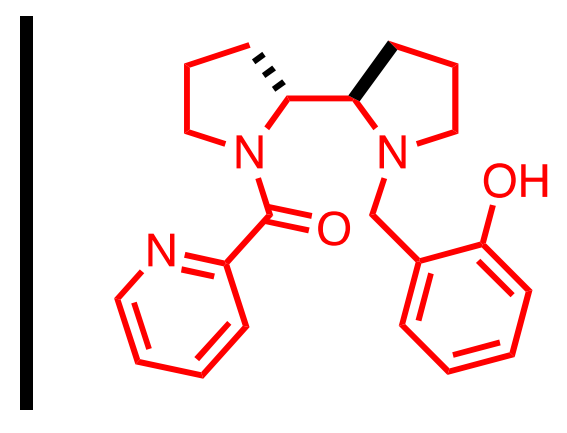



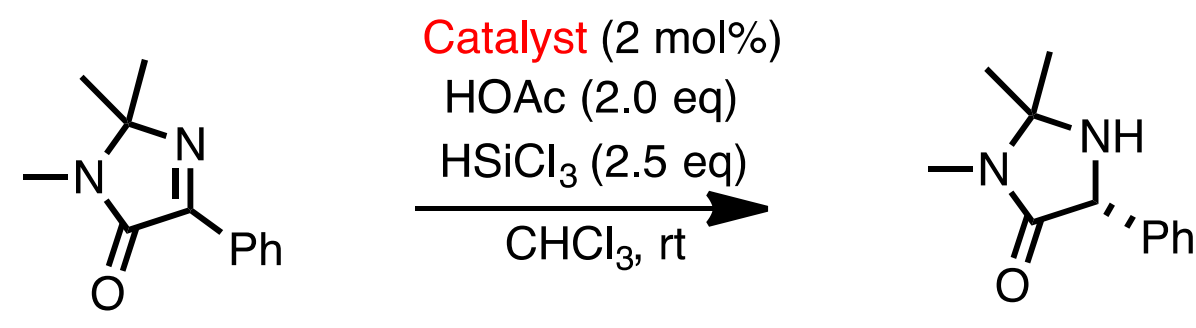

$$
\begin{gathered}
99 \% \text { yield } \\
94 \% \text { ee }
\end{gathered}
$$

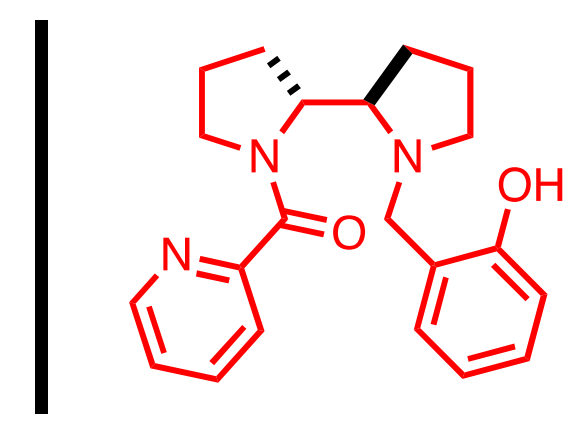

- Mild reaction conditions

- Low catalyst loading

- A broad range of chiral imidazolidinones

- Imidazolinones have to be synthesized by oxidation of racemic imidazolidinones 


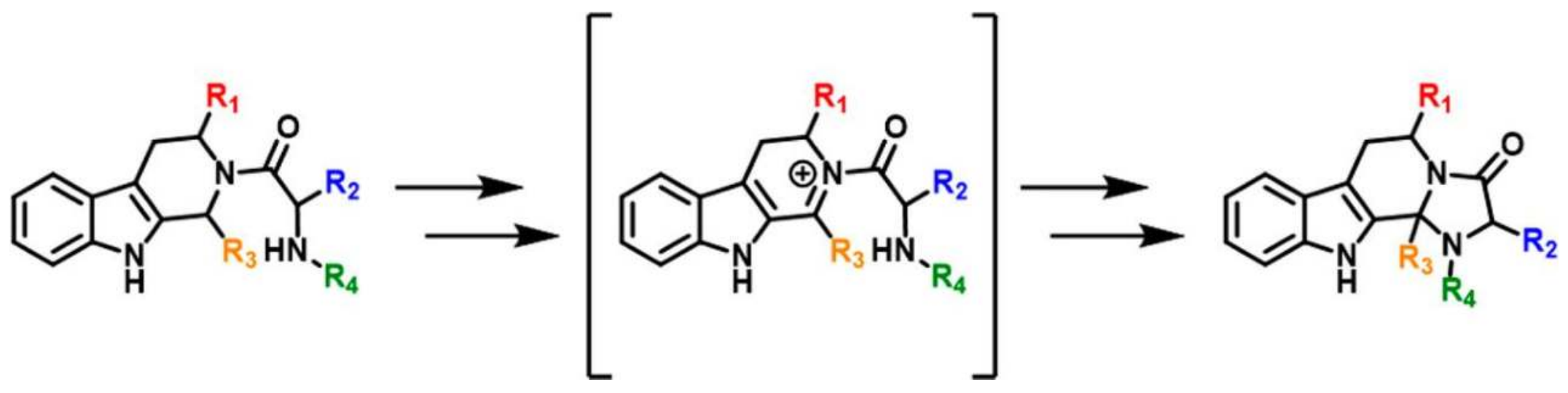

- $\mathrm{N}$-acyliminium formation from readily available precursors and cyclization under mild reaction conditions.

- The diastereoselectivity in the formation of the products is influenced by the specific substituents of the starting reagents.

- However, the method shows moderate or low stereoselectivity 
Synthesis of Polycyclic Imidazolidinones via Amine Redox-Annulation

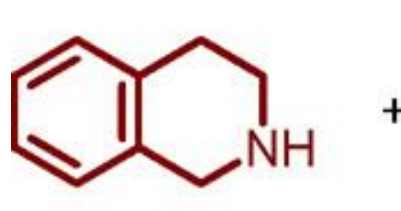<smiles>O=C(Nc1ccccc1)C(=O)c1ccccc1</smiles>
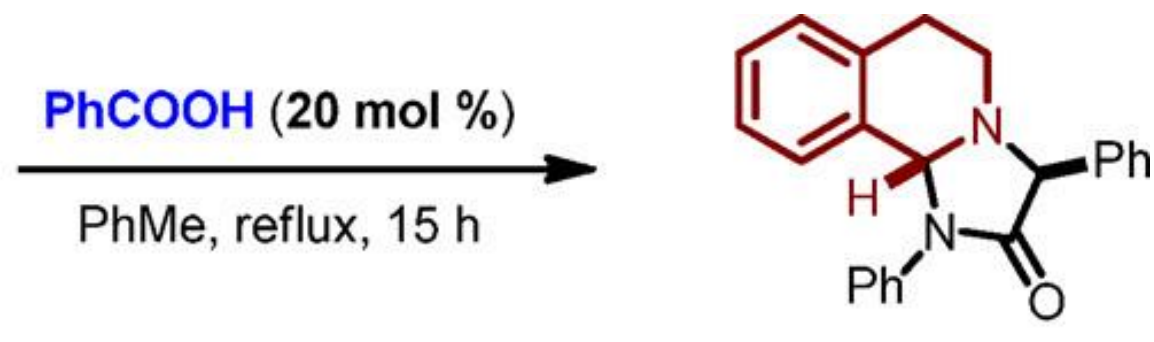

$95 \%$ yield

$\mathrm{dr}>25: 1$ 
Oxidative dearomatization/nucleoophilic nitrogen:

Ciufolini and co: J. Org. Chem., 2000, 65, 4397.
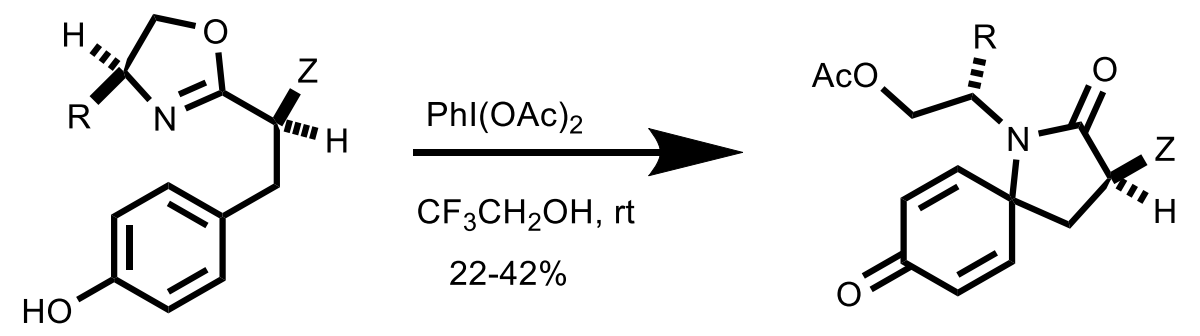

Ciufolini and co: Tetrahedron Lett., 2002, 43, 5193.<smiles>[R][SH](=O)NCCCc1ccc(O)cc1</smiles>
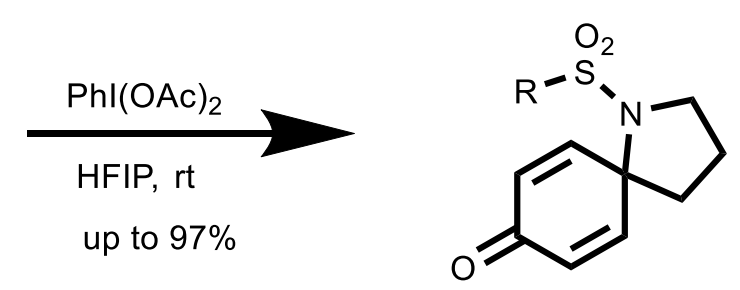

Shunsuke Chiba and co.: JACS., 2010, 132, 7266.
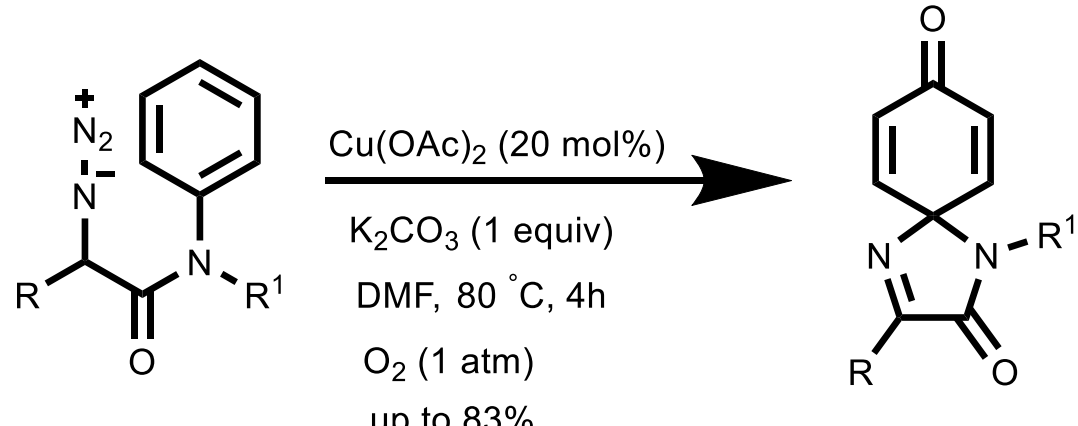

p to $83 \%$ 

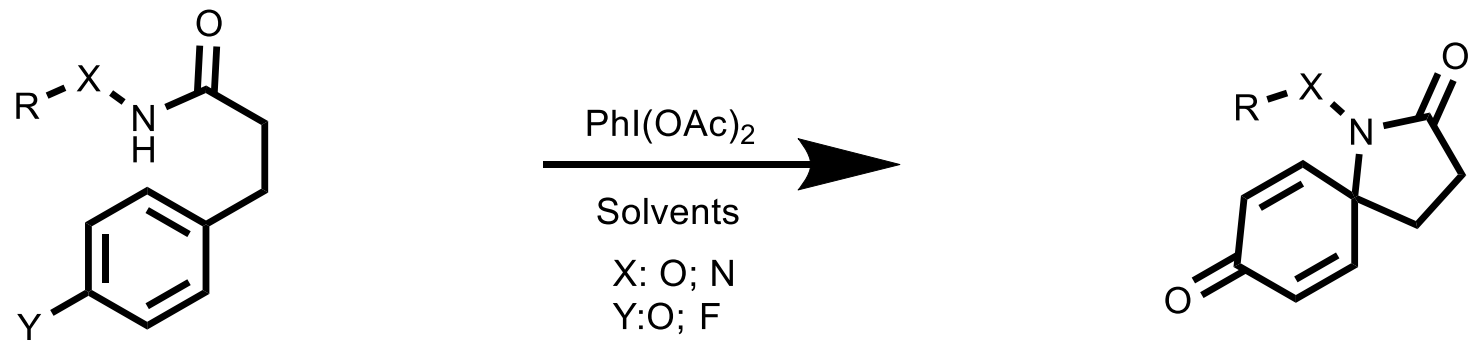

Wardrop and co.: Org. Lett., 2001, 3, 1053;

Org. Lett., 2001, 3, 2353;

Kikugawa and co.: J. Org.Chem., 2003, 68, 5429;

J. Org. Chem., 2003, 68, 6739 


\section{This work: One pot sequence oxidative amidation of aminophenols}
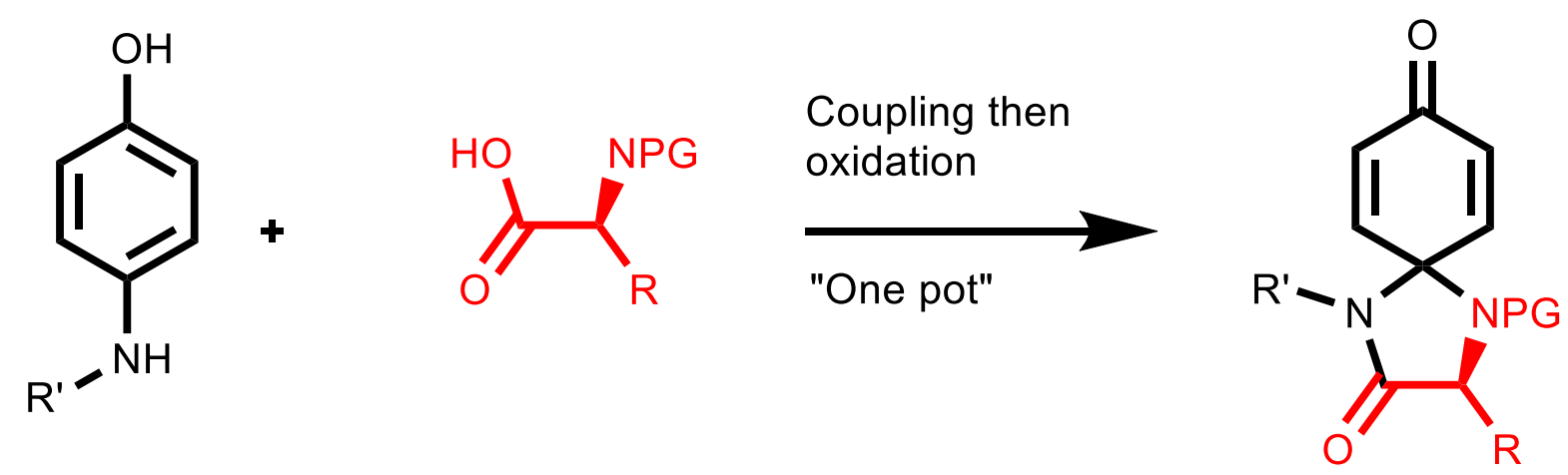

commercially

available a.a chirons

chiral imidazolidinones/ carbamate protected

- New oxidative amidation with N-carbamate amino acid chirons

- One pot reaction

- Fast, efficient and operational simple 


\section{Oxidative Dearomatization of Phenols}
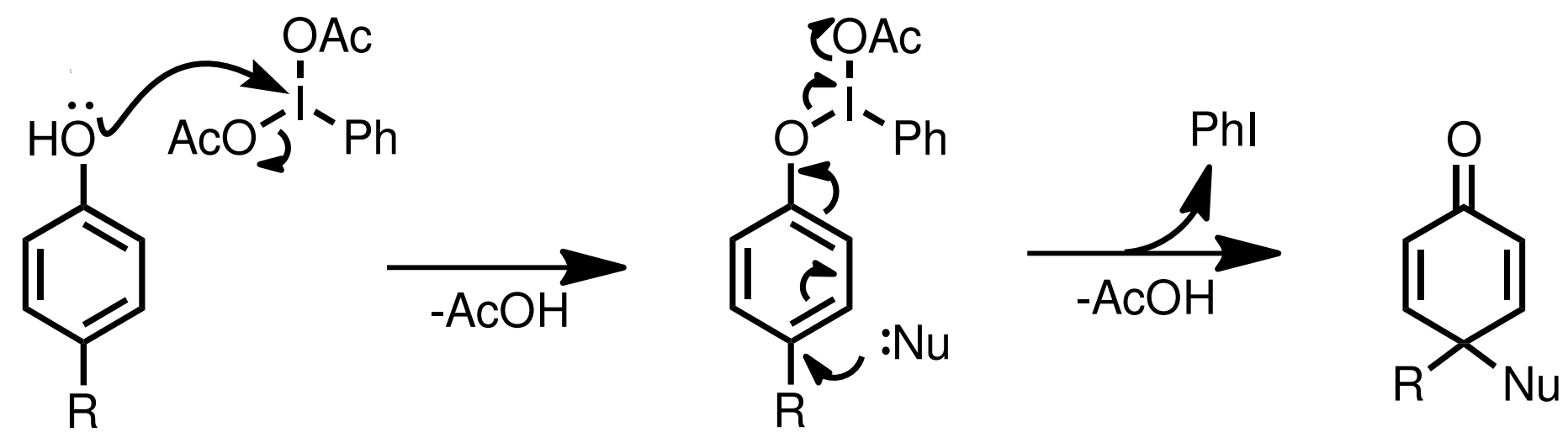

- Mild reaction conditions

- Both intra- and intermolecular reactions work well

- Broad substrate scope

- Useful for functionalization of aromatic structures

Synthesis., 2013, 45, 1-16 

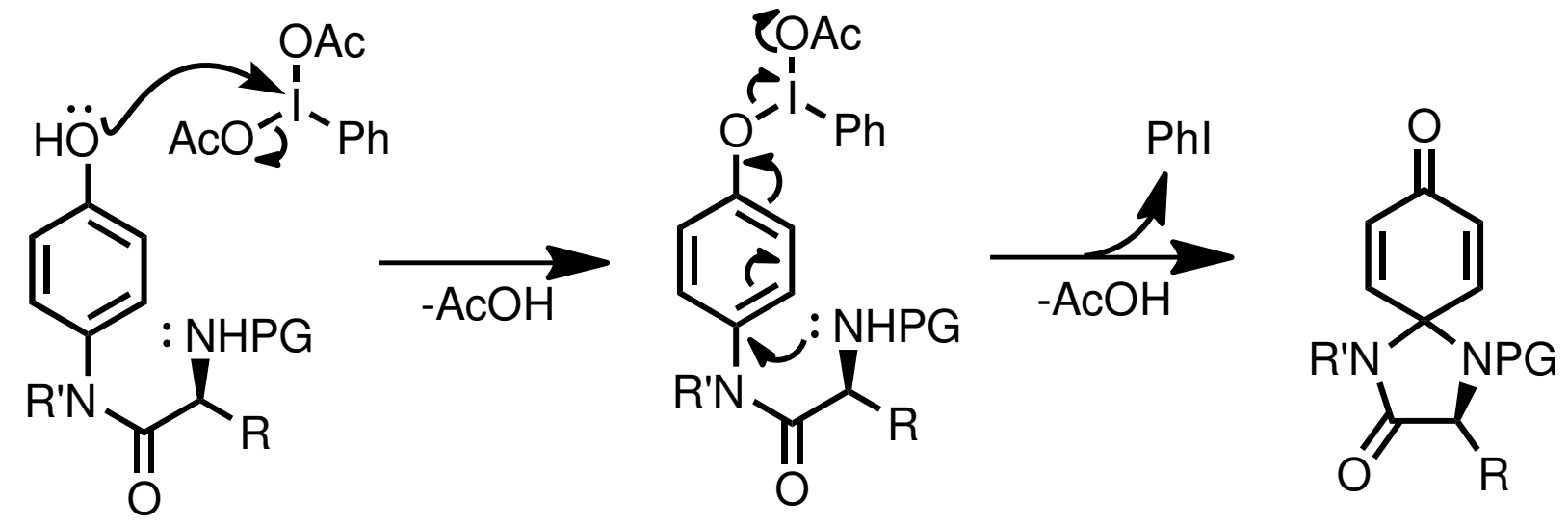

PG: Protecting Groups

- The starting materials, amino amides, derived from natural amino acids and $\mathrm{N}$-alkyl aminophenol, are easy to access.

- The chirality of amino acids is preserved.

- The novel chiral sprioimidazolidinones with many distinctive functional groups could be used as new organocatalysts or as chiral intermediates for construction of complex molecules. 


\section{Oxidative Dearomatization/ipso Cyclization Cascade: Solvents Screening}

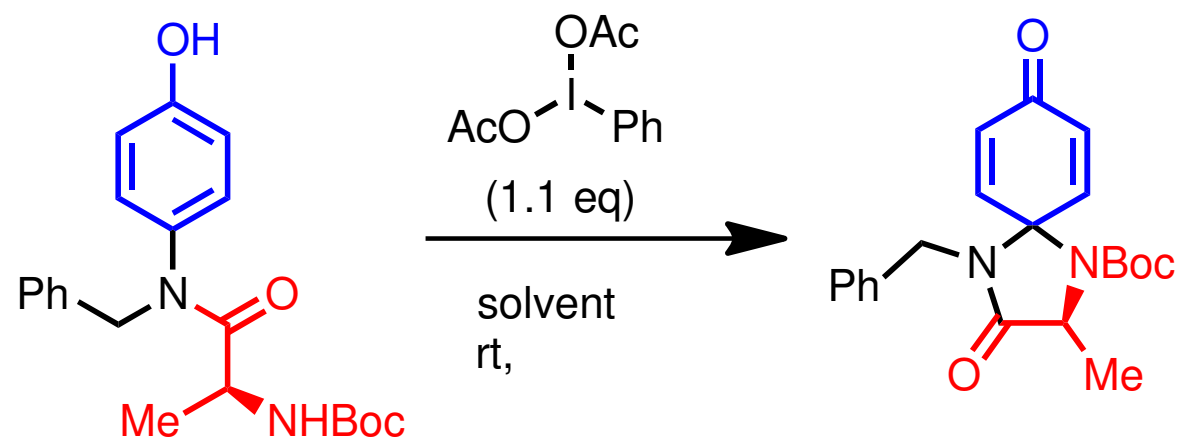

\begin{tabular}{|c|c|c|c|c|}
\hline Entry & Oxidant & Solvent & Additive & Result \\
\hline 1 & $\mathrm{Phl}(\mathrm{OAc})_{2}$ & $\mathrm{Et}_{2} \mathrm{O}$ & None & No desired product \\
\hline 2 & $\mathrm{Phl}(\mathrm{OAc})_{2}$ & $\mathrm{CH}_{2} \mathrm{Cl}_{2}$ & None & No desired product \\
\hline 3 & $\mathrm{Phl}(\mathrm{OAc})_{2}$ & $\mathrm{CHCl}_{3}$ & None & No desired product \\
\hline 4 & $\mathrm{Phl}(\mathrm{OAc})_{2}$ & THF & None & No desired product \\
\hline 5 & $\mathrm{Phl}(\mathrm{OAc})_{2}$ & $\mathrm{C}_{6} \mathrm{H}_{5} \mathrm{CH}_{3}$ & None & No desired product \\
\hline 6 & $\mathrm{Phl}(\mathrm{OAc})_{2}$ & $\mathrm{CF}_{3} \mathrm{CH}_{2} \mathrm{OH}$ & None & $90 \%$ \\
\hline 7 & $\mathrm{Phl}(\mathrm{OAc})_{2}$ & $\mathrm{CH}_{3} \mathrm{OH}$ & None & No desired product \\
\hline 8 & $\mathrm{Phl}(\mathrm{OAc})_{2}$ & $\mathrm{CH}_{3} \mathrm{CN}$ & None & $35 \%$ \\
\hline 9 & $\mathrm{Phl}(\mathrm{OAc})_{2}$ & DMF & None & $25 \%$ \\
\hline 10 & IBX & $\mathrm{CF}_{3} \mathrm{CH}_{2} \mathrm{OH}$ & None & No desired product \\
\hline 11 & DMP & $\mathrm{CF}_{3} \mathrm{CH}_{2} \mathrm{OH}$ & None & No desired product \\
\hline 12 & $\mathrm{NaIO}_{4}$ & $\mathrm{CF}_{3} \mathrm{CH}_{2} \mathrm{OH}$ & None & No reaction \\
\hline 13 & $\mathrm{Phl}(\mathrm{OAc})_{2}$ & $\mathrm{CF}_{3} \mathrm{CH}_{2} \mathrm{OH}$ & Urea & $90 \%$ \\
\hline 14 & $\mathrm{Phl}(\mathrm{OAc})_{2}$ & TFE: $\mathrm{CH}_{3} \mathrm{CN}(3: 1)$ & Urea & $87 \%$ \\
\hline 15 & $\mathrm{Phl}(\mathrm{OAc})_{2}$ & TFE: $\mathrm{CH}_{3} \mathrm{CN}(2: 1)$ & Urea & $80 \%$ \\
\hline 16 & $\mathrm{Phl}(\mathrm{OAc})_{2}$ & TFE: $\mathrm{CH}_{3} \mathrm{CN}(1: 1)$ & Urea & $65 \%$ \\
\hline
\end{tabular}




\section{Optimized condition for one pot procedure}

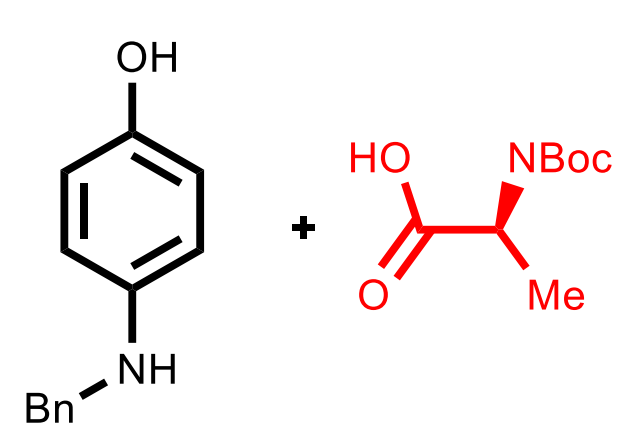

DCC (1 equiv) $/ \mathrm{CH}_{3} \mathrm{CN}$ (1V)

$0{ }^{\circ} \mathrm{C}-\mathrm{rt}, 2 \mathrm{~h}$

$\mathrm{CF}_{3} \mathrm{CH}_{2} \mathrm{OH}(3 \mathrm{~V})$

$\mathrm{Phl}(\mathrm{OAc})_{2}$ (1.0 equiv), $5 \mathrm{~min}$

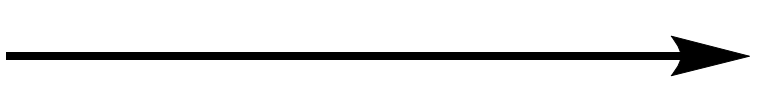

$65 \%$

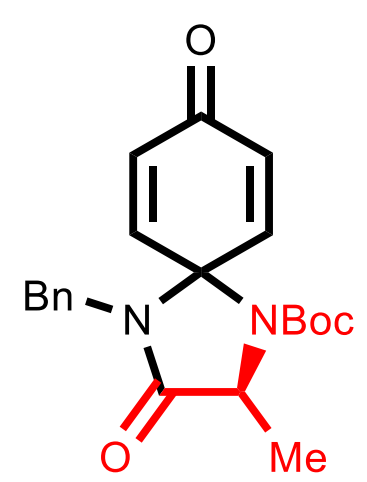


One-pot procedure: the synthesis of chiral spiroimidazolidinones

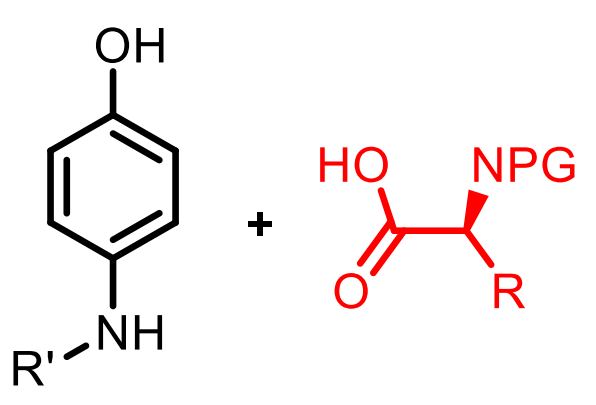

DCC (1 equiv) $/ \mathrm{CH}_{3} \mathrm{CN}$

$0{ }^{\circ} \mathrm{C}-\mathrm{rt}, 2 \mathrm{~h}$

$\mathrm{CF}_{3} \mathrm{CH}_{2} \mathrm{OH}$

$\mathrm{Phl}(\mathrm{OAc})_{2}$ (1.0 equiv), 5 min
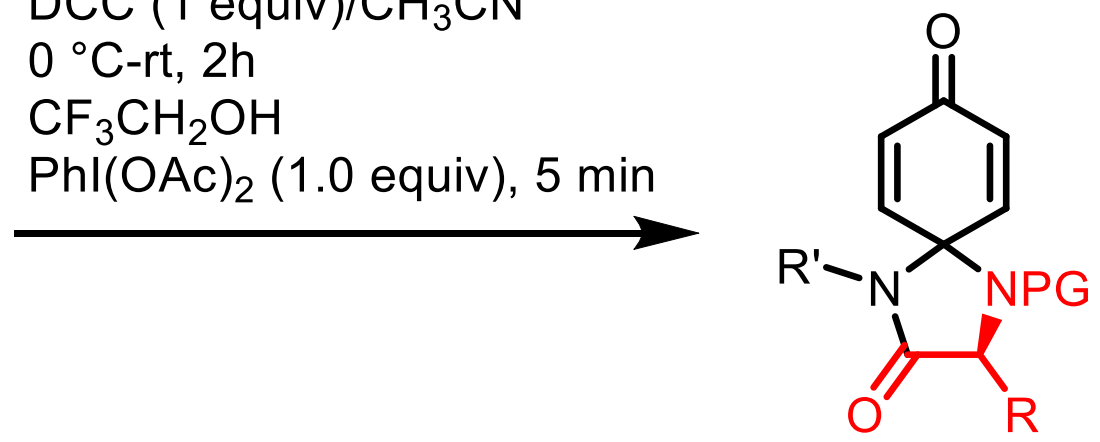


\section{Reaction scope}
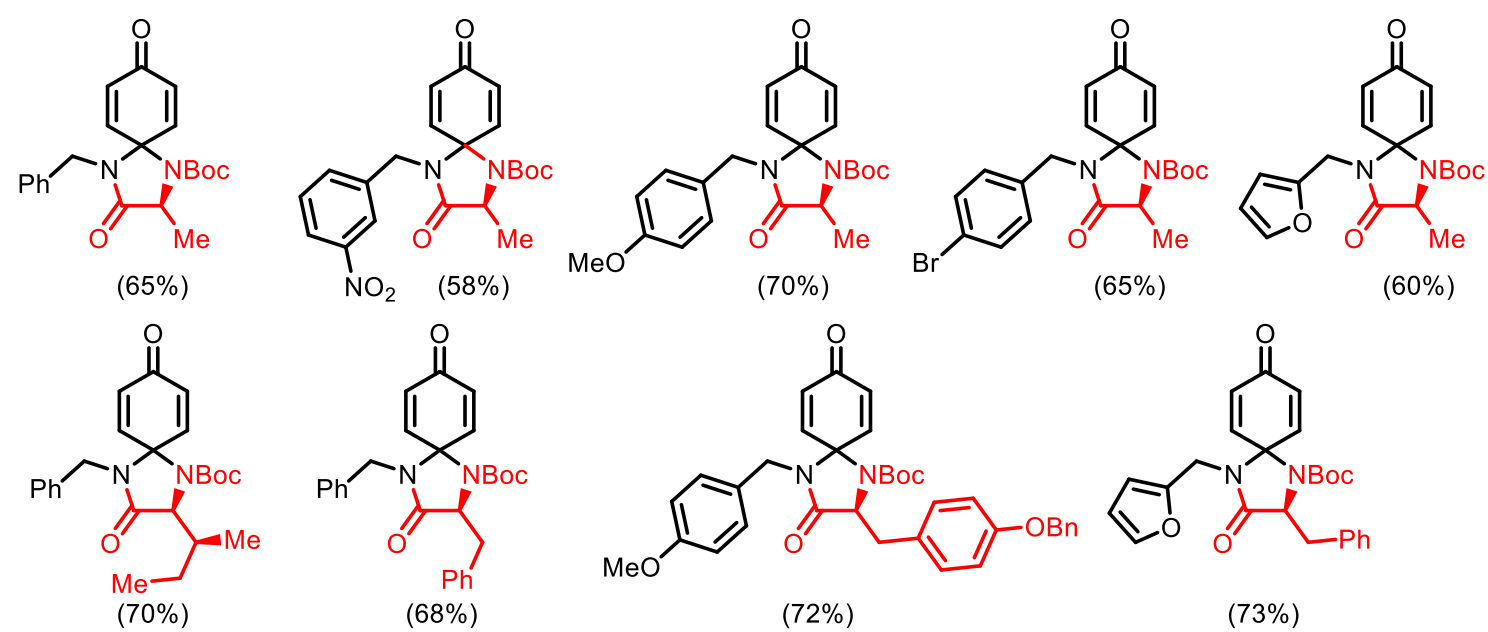

(73\%)
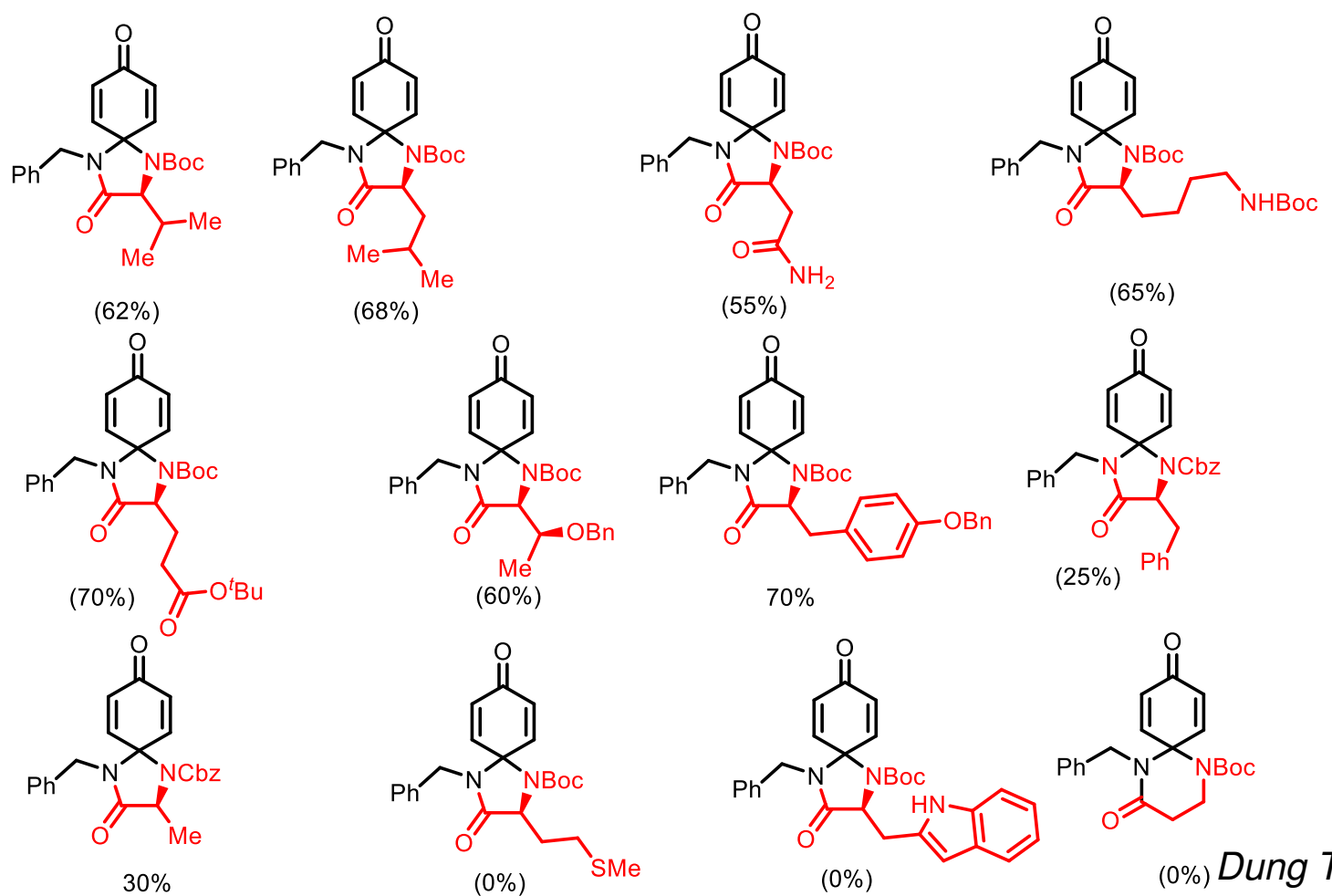


\section{Large Scale Preparation}

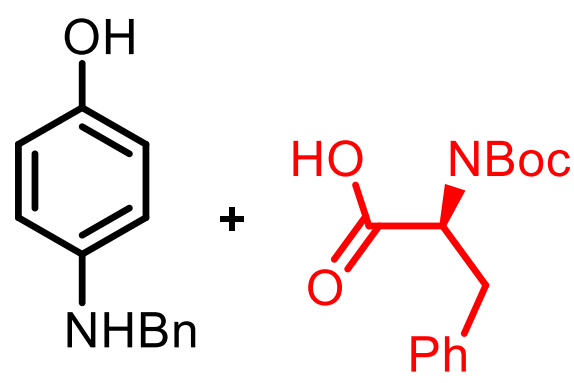

DCC (1 equiv)

$\mathrm{CH}_{3} \mathrm{CN}$

$0{ }^{\circ} \mathrm{C}-\mathrm{rt}, 8 \mathrm{~h}$

$\mathrm{CF}_{3} \mathrm{CH}_{2} \mathrm{OH}$

$\mathrm{Phl}(\mathrm{OAc})_{2}$ (1.0 equiv)

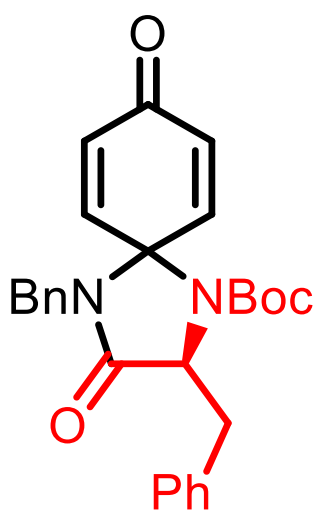

- $20 \mathrm{mmol}$ scale

- $65 \%$ yield

- $5.7 \mathrm{~g}$ final product 


\section{X-Ray Diffraction}

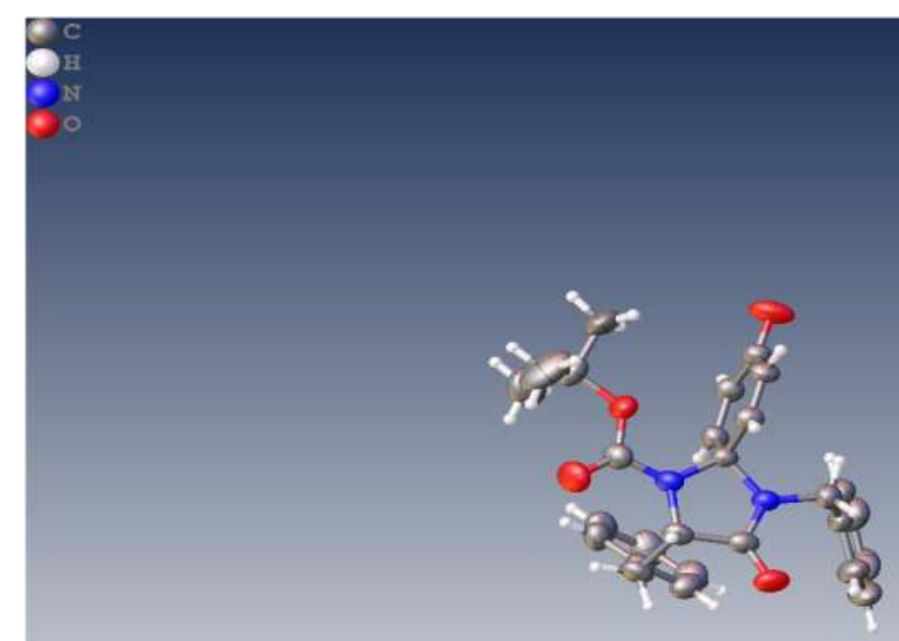<smiles>[B]N1C(=O)[C@@H]([R6](=O)(O)OC(C)(C)C)C(Cc2ccccc2)C12C=CC(=O)C=C2</smiles>

- The structure of $X$ was unambiguously confirmed by $X$-ray crystal structure. As depicted in the $X$-ray diffraction picture, one of the alkene protons in the cyclohexadienone ring (the $\beta$ - $\mathrm{CH}$ of the carbonyl) positions right over the shielding region of the phenyl ring original from phenylalanine. This special feature results in a big shift to the strong field for this proton in $1 \mathrm{H}-\mathrm{NMR}$ (to 4.5ppm). These abnormal phenomena were also observed in the products with an aromatic group in the side chain of the original amino acids (entry). In cases where no such a phenyl group presence, a normal chemical shift for those alkene protons was observed 


\section{Cross over control experiment}

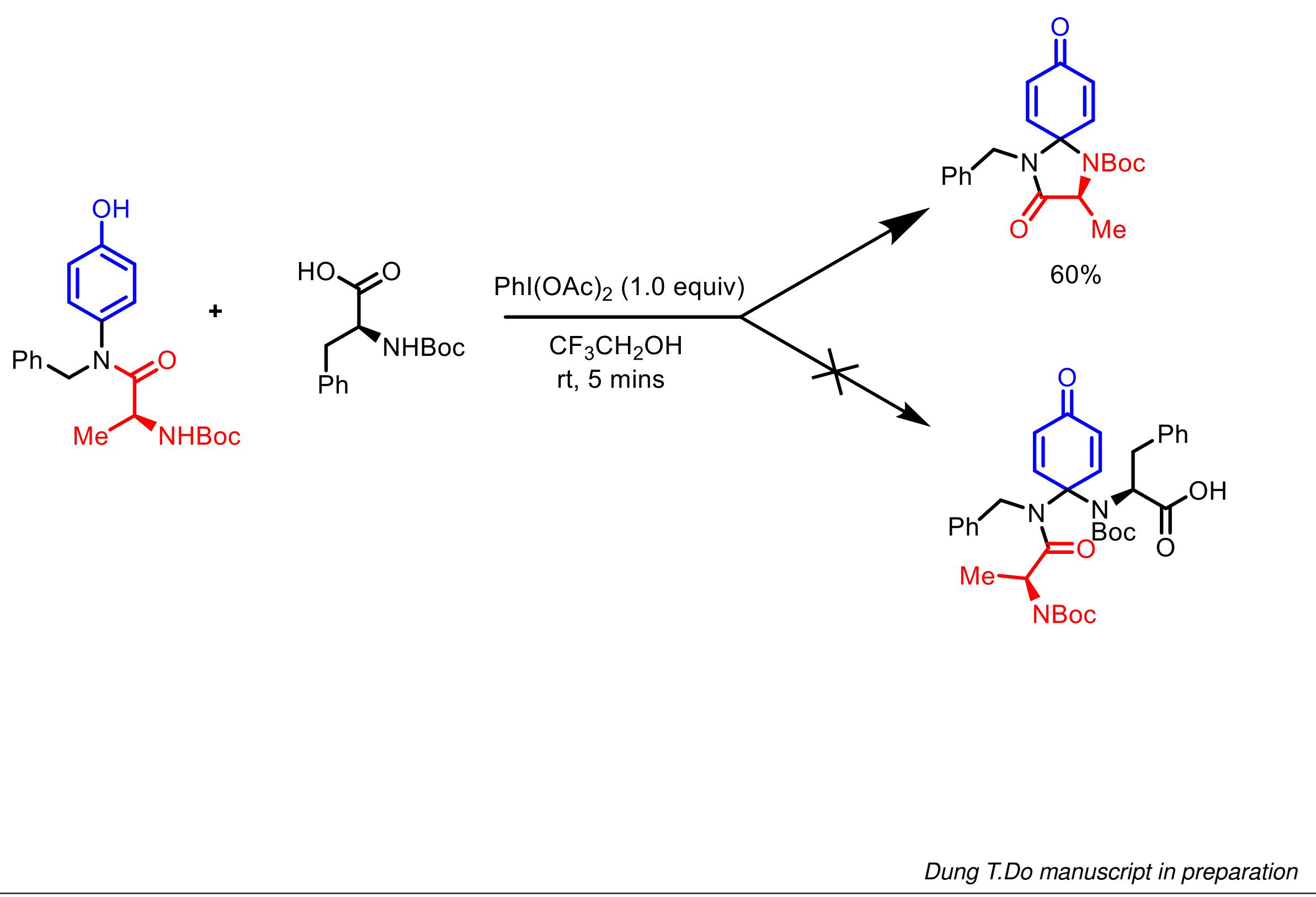




\section{Derivatization of substrates}<smiles></smiles><smiles>CC(C)(C)O[13C](=O)[C@@H](Cc1ccccc1)C12C=CC(=O)C=C1N2Cc1ccccc1</smiles>

$\mathrm{PhB}(\mathrm{OH})_{2}$ (1.5 equiv)

$\mathrm{Pd}\left(\mathrm{PPh}_{3}\right)_{3} \mathrm{Cl}_{2}(5.0 \mathrm{~mol} \%)$

$\mathrm{K}_{3} \mathrm{PO}_{4}$ (3.0equiv)

dioxane $/ \mathrm{H}_{2} \mathrm{O} 2: 1$

microwave, $90{ }^{\circ} \mathrm{C}, 2 \mathrm{~h}$

1.TFA

rt, $3 \mathrm{~h}$

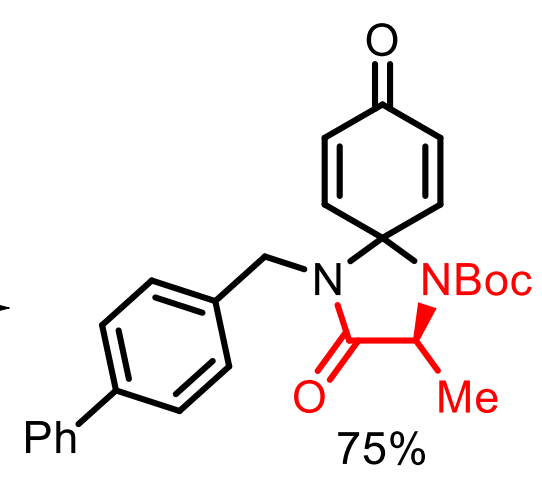<smiles>O=C1C=CC2(C=C1)N[C@H](Cc1ccccc1)C(=O)N2Cc1ccccc1</smiles>

$70 \%$ 


\section{Conclusion}
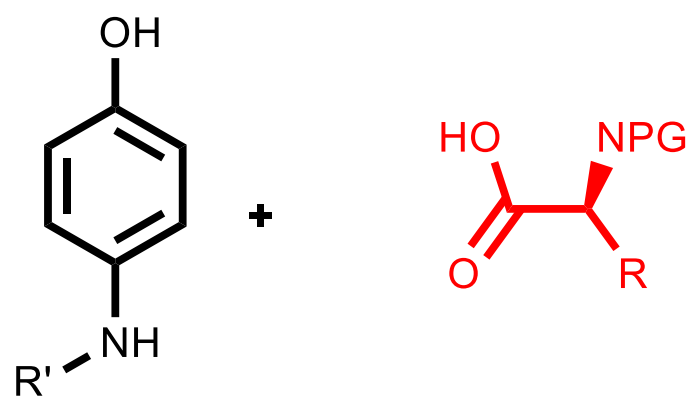

DCC (1 equiv)/ $\mathrm{CH}_{3} \mathrm{CN}$

$$
0{ }^{\circ} \mathrm{C}-\mathrm{rt}, 2 \mathrm{~h}
$$

$\mathrm{CF}_{3} \mathrm{CH}_{2} \mathrm{OH}$

$\mathrm{Phl}(\mathrm{OAc})_{2}$ (1.0 equiv), $5 \mathrm{~min}$

commercially

available a.a chirons

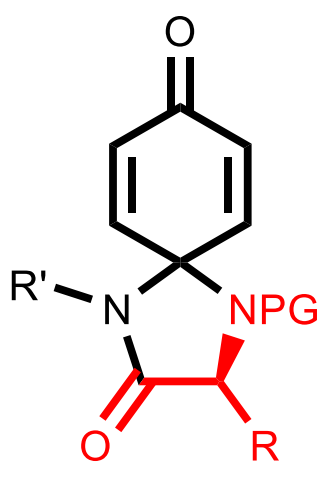

chiral imidazolidinones/ carbamate protected

- New oxidative amidation with $\mathrm{N}$-carbamate amino acid chirons

- One pot reaction

- Fast, efficient and operational simple

- Large scale preparation 


\section{Acknowledgments}

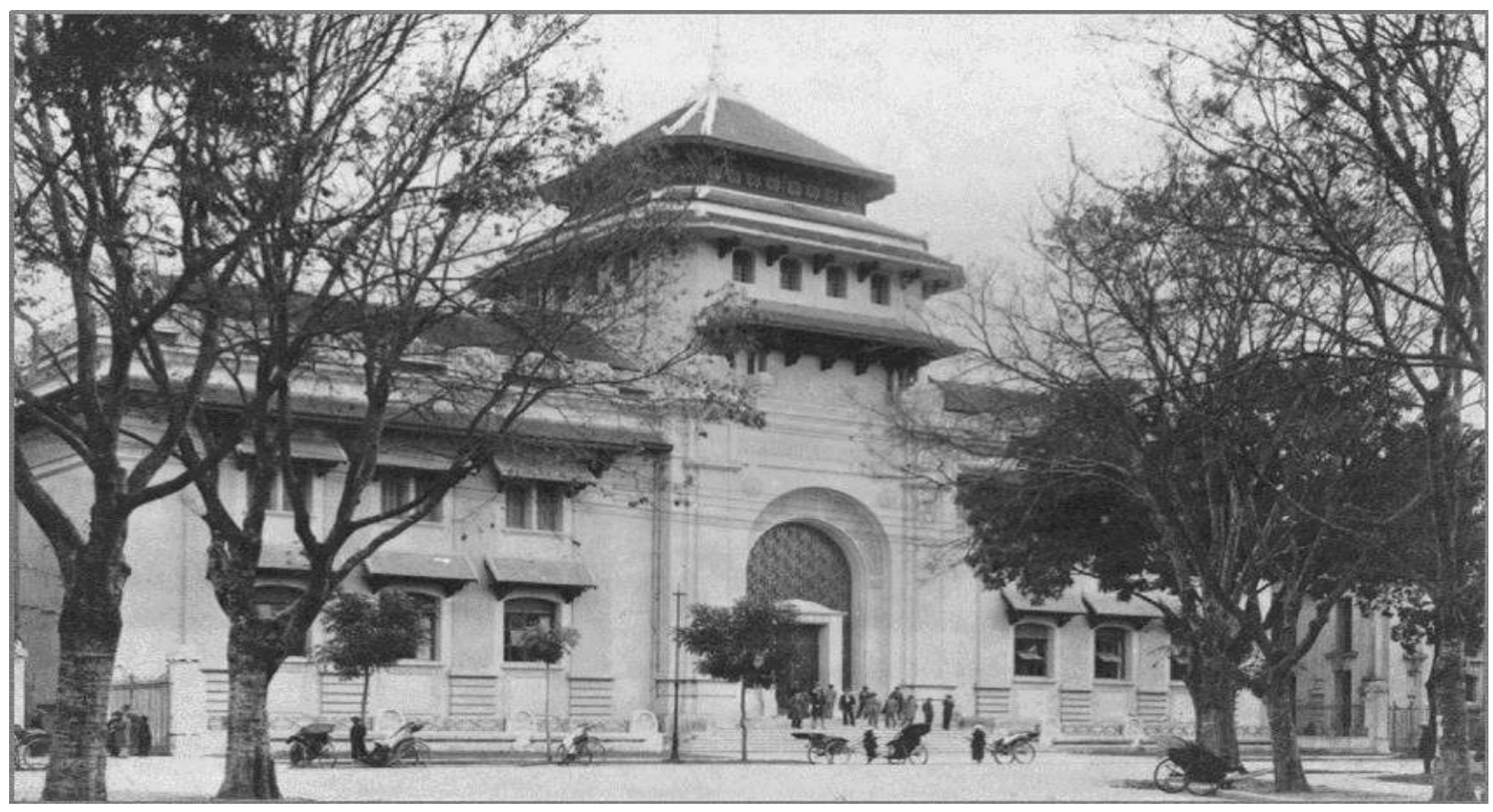

VNU Department of Chemistry

Mac Group for sharing facility 